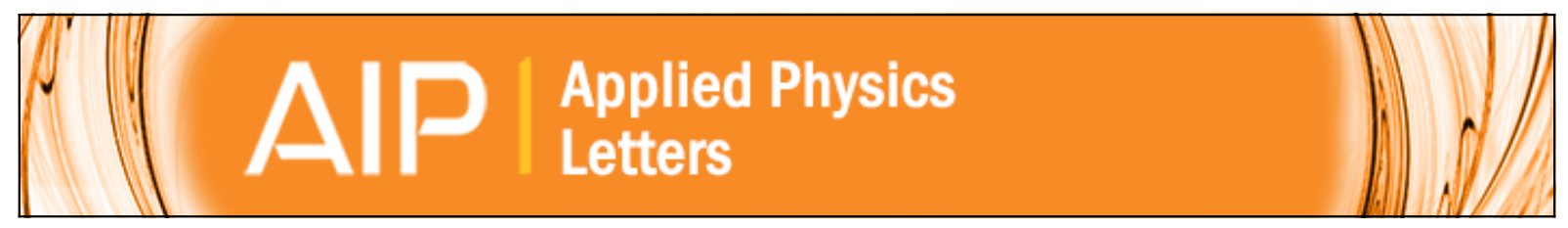

Perpendicular exchange bias in antiferromagnetic-ferromagnetic nanostructures

J. Sort, B. Dieny, M. Fraune, C. Koenig, F. Lunnebach, B. Beschoten, and G. Güntherodt

Citation: Applied Physics Letters 84, 3696 (2004); doi: 10.1063/1.1737484

View online: http://dx.doi.org/10.1063/1.1737484

View Table of Contents: http://scitation.aip.org/content/aip/journal/apl/84/18?ver=pdfcov

Published by the AIP Publishing

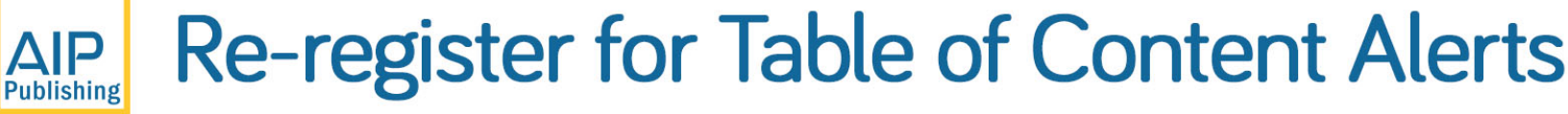

\section{Create a profile. \\ Sign up today!}




\title{
Perpendicular exchange bias in antiferromagnetic-ferromagnetic nanostructures
}

\author{
J. Sort ${ }^{\mathrm{a})}$ and B. Dieny \\ SPINTEC (URA 2512 CNRS/CEA), CEA/Grenoble, 17 Av. Martyrs, 38054 Grenoble Cedex 9, France \\ M. Fraune, C. Koenig, F. Lunnebach, B. Beschoten, and G. Güntherodt \\ II. Physikalisches Institut, RWTH Aachen, 52056 Aachen, Germany
}

(Received 21 November 2003; accepted 16 March 2004; published online 20 April 2004)

\begin{abstract}
Exchange bias effects have been induced along the perpendicular-to-film direction in nanostructures prepared by electron beam lithography, consisting of a ferromagnetic $[\mathrm{Pt} / \mathrm{Co}]$ multilayer exchange coupled to an antiferromagnet (FeMn). As a general trend, the exchange bias field and the blocking temperature decrease, whereas the coercivity increases, as the size of the nanostructures is reduced.

(C) 2004 American Institute of Physics. [DOI: 10.1063/1.1737484]
\end{abstract}

During the last decades, the areal density of data storage devices has dramatically increased each year. This trend demands a miniaturization of the reading/writing magnetic data devices. Reading heads are typically composed of spin-valve or tunnel junction structures, in which antiferromagnetic $(\mathrm{AFM}) /$ ferromagnetic $(\mathrm{FM})$ exchange biased bilayers constitute an essential part ${ }^{1}$ (for recent reviews on exchange bias, see Refs. 2-5). It is worth noting that, during the last years, the exchange bias effects arising from reduced dimensions have been investigated to some extent. ${ }^{6-12}$ However, so far, these studies have only been carried out in systems with an in-plane magnetic anisotropy.

Recently, it has been shown that it is also possible to induce exchange bias in continuous multilayers exhibiting a perpendicular-to-film magnetic anisotropy. ${ }^{13-15}$ Perpendicular exchange bias offers the possibility of preparing spin valves or tunnel junctions with perpendicular magnetization. ${ }^{16-18}$ It should be noted that in order to achieve dense packing of particles (or nanodevices), nanostructures with perpendicular anisotropy are preferable. ${ }^{19-21}$

In this letter, we demonstrate that it is possible to induce perpendicular exchange bias in nanostructures consisting of a [Pt/Co] multilayer (ML) exchange coupled to FeMn. Our results show that the magnitude of exchange bias decreases as the lateral size of the nanostructure is reduced. Moreover, the temperature at which exchange bias disappears during heating also decreases when the continuous film is patterned.

A continuous multilayer with the composition $[\operatorname{Pt}(20 \AA) / \operatorname{Co}(4 \AA)]_{5} / \operatorname{Pt}(5 \AA) / \operatorname{FeMn}(130 \AA) / \operatorname{Pt}(20 \AA)$ was deposited onto a thermally oxidized $\mathrm{Si}$ wafer by dc magnetron sputtering. From the sputtered multilayer, different types of nanostructures were fabricated by electron beam lithography (for a recent review on fabrication and properties of magnetic ordered arrays of nanostructures, see Ref. 22). Shown in Fig. 1 are the scanning electron microscopy images of (a) the $200 \times 200 \mathrm{~nm}^{2}$ square dots, (b) the $200 \mathrm{~nm}$ $\times 1 \mu \mathrm{m}$ stripes and (c) the $200 \mathrm{~nm} \times 100 \mu \mathrm{m}$ wires. The continuous film and the nanostructures were field cooled under a

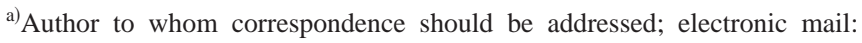
jordi.sort@uab.es
}

field of $2.5 \mathrm{kOe}$, applied perpendicular to the film plane, from $T=600 \mathrm{~K}$. Hysteresis loops were measured by polar Kerr effect.

Figure 2 shows the hysteresis loops of (a) the continuous film, (b) the 200-nm-wide $\times 100-\mu \mathrm{m}$-long wires, (c) the $200 \mathrm{~nm} \times 1 \mu \mathrm{m}$ stripes and (d) the $200 \times 200 \mathrm{~nm}^{2}$ dots. All the hysteresis loops are rather square, with a remanence to saturation ratio, $M_{R} / M_{S}$, close to 1 . In arrays of very small dots, it is rather common to observe a broad distribution of switching fields. ${ }^{22}$ This probably accounts for the slight tilt of the hysteresis loop observed in the $200 \times 200 \mathrm{~nm}^{2}$ dots. The good squareness of the loops indicates that the perpendicular effective magnetic anisotropy is essentially maintained during the patterning process. Since the perpendicular anisotropy in [Pt/Co] ML arises from the interface anisotropy between the Pt and Co layers, ${ }^{23}$ it is likely that the Pt/Co interfaces remain parallel to the thin film plane after the patterning.

The magnitudes of the coercivity, $H_{C}$, and the exchange bias field, $H_{E}$, for the continuous film and the different nanostructures investigated are shown in Table I. It is worth noting that in the $200 \times 200 \mathrm{~nm}^{2}$ dots $H_{C}$ increases by a factor of 4 compared to the continuous film. On the contrary, $H_{E}$ decreases from 223 Oe (in the continuous film) to about 14 Oe (in the smallest dots). The enhancement of $H_{C}$ is commonly observed in patterned elements and is probably due to the role of the edges of the nanostuctures as barriers for the domain wall propagation. The decrease of $H_{E}$ as the size of the nanostructure is reduced has also been sometimes observed in AFM-FM patterned elements with in-plane magnetic anisotropy. ${ }^{7,8,10}$ This decrease can be attributed to the constraints imposed by the reduced dimensions of the nanostructures on the formation of antiferromagnetic domain walls. Indeed, the majority of models dealing with exchange bias relate the magnitude of $H_{E}$ to the formation of domains in the AFM layer. ${ }^{24-26}$ It can be argued that the existence of AFM domain walls allows a small surplus of magnetization at the AFM/FM interface, which couples to the FM, resulting in the unidirectional anisotropy, i.e., exchange bias. In fact, taking into account the values of the magnetic stiffness for FeMn $\left(A_{\mathrm{FeMn}}=3 \times 10^{-7} \mathrm{erg} / \mathrm{cm}\right)$ and its magnetic anisotropy $\left(K_{\mathrm{FeMn}}=1.3 \times 10^{5} \mathrm{erg} / \mathrm{cm}^{3}\right)$, the domain wall width in 

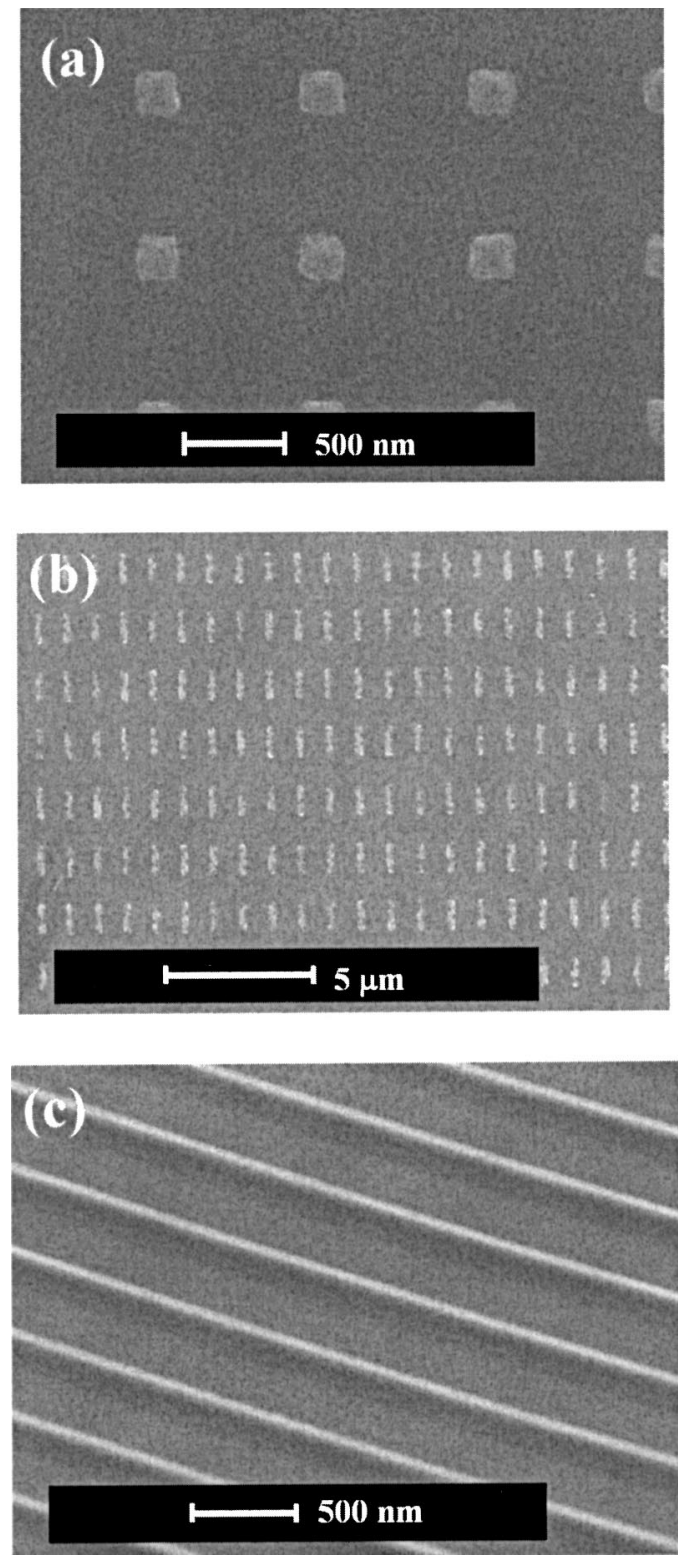

FIG. 1. Scanning electron microscopy (SEM) images of (a) the 200 $\times 200 \mathrm{~nm}^{2}$ square dots, (b) the $200 \mathrm{~nm} \times 1 \mu \mathrm{m}$ stripes, and (c) the $200 \mathrm{~nm}$ $\times 100 \mu \mathrm{m}$ wires, which consist of a multilayer system with the composition $[\operatorname{Pt}(20 \AA) / \operatorname{Co}(4 \AA)]_{5} / \operatorname{Pt}(5 \AA) / F e M n(130 \AA) / \operatorname{Pt}(20 \AA)$.

FeMn, $\delta_{\mathrm{FeMn}}, \quad$ can be estimated to be $\delta_{\mathrm{FeMn}}$ $=\pi\left(A_{\mathrm{FeMn}} / K_{\mathrm{FeMn}}\right)^{1 / 2} \sim 50 \mathrm{~nm} .{ }^{24}$ When the lateral dimensions of the nanostructures become about the same order of magnitude as the AFM domain wall width, it is likely that some AFM domain walls, instead of being able to completely form may be just partially developed inside the nanostructures. These partial AFM domain walls may be less effectively pinned than complete $180^{\circ}$ AFM domain walls in continuous AFM-FM films, hence leading to the observed decrease of $H_{E}$ in the nanostructures. It should be noted that our results are opposite to a theoretical study ${ }^{27}$ which predicted an $H_{E}$ enhancement in AFM/FM nanostructures with relatively large AFM anisotropies and an experimental study $^{28}$ where an $H_{E}$ enhancement was observed in FM dots on macroscopic AFM.

Furthermore, it is well known that FM particles tend to lose their magnetic order when their size becomes very small. A reduction of the Curie temperature in ultrafine FM

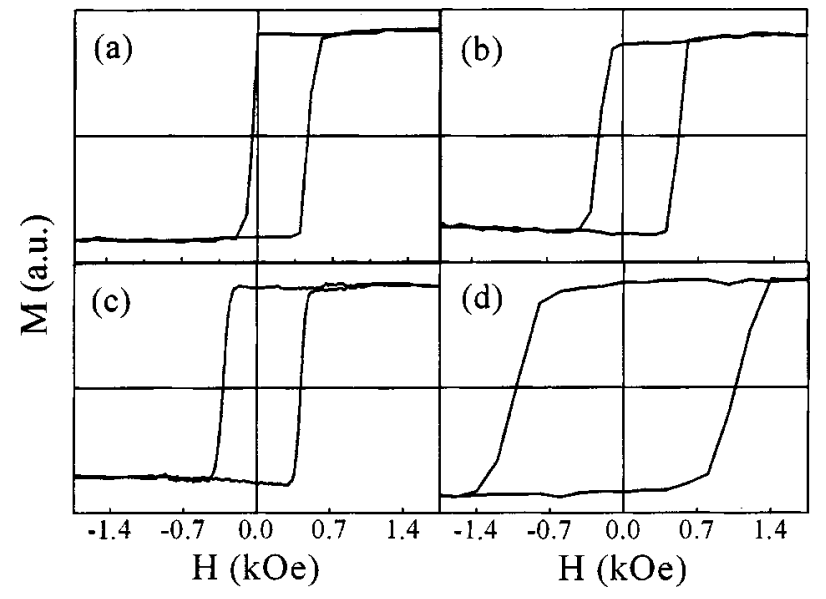

FIG. 2. Hysteresis loops measured at room temperature by polar Kerr effect, of (a) the continuous multilayer, (b) the 200-nm-wide nanowires, (c) the $200 \mathrm{~nm} \times 1 \mu \mathrm{m}$ stripes, and (d) the $200 \times 200 \mathrm{~nm}^{2}$ dots.

particles has been experimentally reported ${ }^{29}$ and theoretically interpreted. ${ }^{30}$ In addition, a decrease of the Néel temperature has also been observed in ultrathin AFM layers or ultrafine AFM particles. ${ }^{31,32}$ By analogy, it could be argued that in AFM-FM systems, a reduction in the temperature at which exchange bias disappears during heating (i.e., the so-called blocking temperature, $T_{B}$ ), may be expected if the continuous bilayer is patterned. Indeed, $T_{B}$ has been found to reduce in AFM-FM continous bilayers with very thin AFM layer thickness or very small AFM crystallites. ${ }^{33,34}$ The temperature dependence of $H_{E}$ is shown in Fig. 3(a). As expected, in both the continuous film and the nanowires, $H_{E}$ decreases during heating due to the loss of magnetic order in the AFM. Nevertheless, $H_{E}$ vanishes in the nanowires at a lower temperature $\left(T_{B}=540 \mathrm{~K}\right)$ than in the continuous films (where $T_{B}=570 \mathrm{~K}$ ). This can be ascribed to an increasing thermally induced unpinning of antiferromagnetic domain walls as the size of the nanostructure is reduced. Moreover, it is remarkable that the overall shapes of the $H_{E}$ vs $T$ curves in the continuous films and the nanowires are quite different. Namely, although in the continuous films this dependence is roughly linear, in the nanowires $H_{E}$ decreases slowly for $T<400 \mathrm{~K}$ and at a higher rate for larger temperatures. This probably indicates that the distribution of local blocking temperatures in the nanostructures is narrower than in the continuous film. In particular, it can be inferred that, during the patterning, those AFM-FM regions of the continuous films with lower local $T_{B}$ may become, in fact, essentially uncoupled.

Figure 3(b) shows the dependence of $H_{C}$ on temperature for the continuous film and the nanowires. Similar to $H_{E}$,

TABLE I. Summary of the values of the exchange bias field, $H_{E}$, and the coercivity, $H_{C}$, evaluated at room temperature, for the continuous film and the different nanostructures investigated in our study.

\begin{tabular}{ccc}
\hline \hline & $H_{C}( \pm 5$ Oe $)$ & $H_{E}( \pm 5$ Oe $)$ \\
\hline Continuous film & 252 & 223 \\
Nanowires $200 \mathrm{~nm} \times 100 \mu \mathrm{m}$ & 368 & 138 \\
Stripes $200 \mathrm{~nm} \times 2 \mu \mathrm{m}$ & 370 & 54 \\
Stripes $200 \mathrm{~nm} \times 1 \mu \mathrm{m}$ & 409 & 51 \\
Dots $200 \mathrm{~nm} \times 200 \mathrm{~nm}$ & 1042 & 14 itation.aip.orgaded to IP: \\
\hline \hline
\end{tabular}



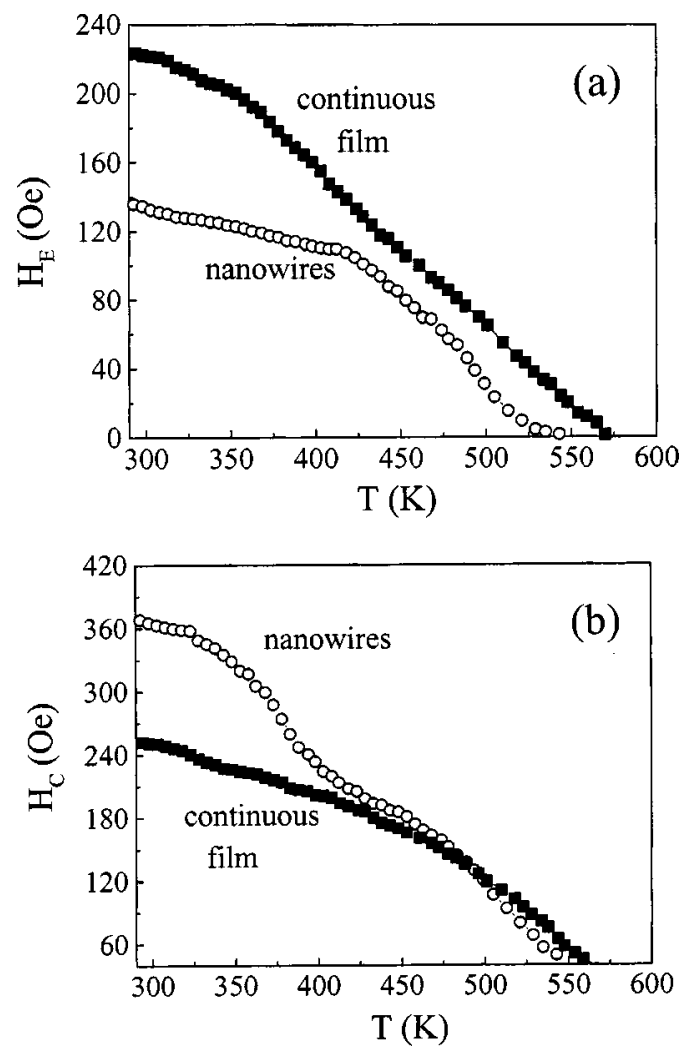

FIG. 3. Dependence on temperature of (a) the exchange bias field, $H_{E}$, and (b) the coercivity, $H_{C}$, for the continuous multilayer film and the patterned 200-nm-wide nanowires.

the coercivity also decreases during heating. Interestingly, although $H_{C}$ is larger in the nanowires at room temperature, the values of $H_{C}$ for the continuous film and the nanowires become similar for $T>450 \mathrm{~K}$ and even slightly smaller in the nanostructures at higher temperatures, where exchange bias virtually vanishes. In fact, one finds that the difference $H_{C}(550 \mathrm{~K})-H_{C}(300 \mathrm{~K})$ is equal to 200 Oe for the continuous film, whereas it is equal to 320 Oe for the nanowires. This is an indication that size effects on AFM/FM coupling are partially responsible for the large $H_{C}$ observed in the nanostructures. In addition, the decrease of $H_{C}$ with temperature is also associated with the characteristic loss of the perpendicular anisotropy in $[\mathrm{Pt} / \mathrm{Co}]$ multilayers. From Fig. 3(b) it seems that the FM anisotropy decreases more rapidly with temperature in the nanostructures than in the continuous films.

Finally, although structural changes at the AFM-FM interface or in the bulk of the AFM due to the ion milling process have not been found to be significant in AFM-FM nanostructures with in-plane anisotropy fabricated by electron beam lithography, ${ }^{7}$ some structural effects, particularly edge defects, should not be completely disregarded as partially responsible for the reduction observed in $H_{E}$ or $T_{B}$ or the increase of $H_{C}$.

In conclusion, we demonstrate the possibility of inducing perpendicular exchange bias in nanostructures composed of a $[\mathrm{Pt} / \mathrm{Co}]$ multilayer exchange coupled with an antiferromagnet (FeMn), fabricated by electron beam lithography. It is found that, although the patterned elements preserve the perpendicular magnetic anisotropy of the multilayer, the magnitude of the exchange bias field progressively decreases and the coercivity increases as the size of the nanostructure is reduced, probably due to the constraints imposed by the small lateral dimensions of the nanostructures on the formation of AFM domain walls. In addition, the blocking temperature of the AFM-FM system is also found to decrease after the patterning process. These phenomena are certainly important to take into account in the implementation of magnetic sensor nanodevices based on spin valves or tunnel junctions with perpendicular anisotropy.

This work was supported by the European Community through NEXBIAS Grant No. HPRN-CT-2002-00296.

${ }^{1}$ B. Dieny, V. S. Speriousu, S. S. P. Parkin, B. A. Gurney, D. R. Wilhoit, and D. Mauri, Phys. Rev. B 43, 1297 (1991).

${ }^{2}$ J. Nogués and I. K. Schuller, J. Magn. Magn. Mater. 192, 203 (1999).

${ }^{3}$ A. E. Berkowitz and K. Takano, J. Magn. Magn. Mater. 200, 552 (1999).

${ }^{4}$ R. L. Stamps, J. Phys. D 33, R247 (2000).

${ }^{5}$ M. Kiwi, J. Magn. Magn. Mater. 234, 584 (2001).

${ }^{6}$ Y. Otani, A. Nemoto, S. G. Kim, K. Fukamichi, O. Kitakami, and Y. Shimada, J. Magn. Magn. Mater. 198, 434 (1999).

${ }^{7}$ M. Fraune, U. Rüdiger, G. Güntherodt, S. Cardoso, and P. Freitas, Appl. Phys. Lett. 77, 3815 (2000).

${ }^{8}$ J. Yu, A. D. Kent, and S. S. P. Parkin, J. Appl. Phys. 87, 5049 (2000).

${ }^{9}$ R. E. Dunin-Borkowski, M. R. McCartney, B. Kardynal, M. R. Scheinfein, D. J. Smith, and S. S. P. Parkin, J. Appl. Phys. 90, 2899 (2001).

${ }^{10}$ Y. Shen, Y. Wu, H. Xie, K. Li, J. Qiu, and Z. Guo, J. Appl. Phys. 91, 8001 (2002).

${ }^{11}$ A. Hoffmann, M. Grimsditch, J. E. Pearson, J. Nogués, W. A. A. Macedo, and I. K. Schuller, Phys. Rev. B 67, 220406(R) (2003).

${ }^{12}$ K. Liu, J. Nogués, C. Leighton, H. Masuda, K. Nishio, I. V. Roshchin, and I. K. Schuller, Appl. Phys. Lett. 81, 4434 (2002).

${ }^{13}$ S. Maat, K. Takano, S. S. P. Parkin, and E. E. Fullerton, Phys. Rev. Lett. 87, 087202 (2001).

${ }^{14}$ Ch. Binek, A. Hochstrat, and W. Kleemann, Phys. Status Solidi A 189, 575 (2002).

${ }^{15}$ F. Garcia, J. Moritz, F. Ernult, S. Auffret, B. Rodmacq, B. Dieny, J. Camarero, Y. Pennec, S. Pizzini, and J. Vogel, IEEE Trans. Magn. 38, 2730 (2002).

${ }^{16}$ F. Garcia, F. Fettar, S. Auffret, B. Rodmacq, and B. Dieny, J. Appl. Phys. 93, 8397 (2003)

${ }^{17}$ F. Garcia, J. Sort, B. Rodmacq, S. Auffret, and B. Dieny, Appl. Phys. Lett. 83, 3537 (2003).

${ }^{18}$ N. Nishimura, T. Hirai, A. Koganei, T. Ikeda, and K. Okano, J. Appl. Phys. 91, 5246 (2002).

${ }^{19}$ A. Moser, K. Takano, D. T. Margulies, M. Albrecht, Y. Sonobe, Y. Ikeda, S. Sun, and E. E. Fullerton, J. Phys. D 35, R157 (2000).

${ }^{20}$ K. Y. Guslienko, S. B. Choe, and S. C. Shin, Appl. Phys. Lett. 76, 3609 (2000).

${ }^{21}$ S. Landis, B. Rodmacq, and B. Dieny, Phys. Rev. B 62, 12271 (2000).

${ }^{22}$ J. I. Martín, J. Nogués, K. Liu, J. L. Vincent, and I. K. Schuller, J. Magn. Magn. Mater. 256, 449 (2003).

${ }^{23}$ M. T. Johnson, P. J. H. Bloemen, F. J. A. den Broeder, and J. J. de Vries, Rep. Prog. Phys. 59, 1409 (1996).

${ }^{24}$ D. Mauri, H. C. Siegmann, P. S. Bagus, and E. Kay, J. Appl. Phys. 62, 3047 (1987)

${ }^{25}$ A. P. Malozemoff, Phys. Rev. B 35, 3679 (1987).

${ }^{26}$ P. Miltényi, M. Gierlings, J. Keller, B. Beschoten, G. Güntherodt, U. Nowak, and K. D. Usadel, Phys. Rev. Lett. 84, 4224 (2000).

${ }^{27}$ S. Zhang and Z. Li, Phys. Rev. B 65, 054406 (2001).

${ }^{28}$ K. Liu, S. M. Baker, M. Touminen, T. P. Russell, and I. K. Schuller, Phys. Rev. B 63, 060403(R) (2001).

${ }^{29}$ F. Huang, M. T. Kief, G. J. Mankey, and R. F. Willis, Phys. Rev. B 49, 3962 (1994).

${ }^{30}$ W. H. Zhong, C. Q. Sun, B. K. Tay, S. Li, H. L. Bai, and E. Y. Jiang, J. Phys.: Condens. Matter 14, L399 (2002).

${ }^{31}$ Y. J. Tang, D. J. Smith, B. L. Zink, F. Hellman, and A. E. Berkowitz, Phys. Rev. B 67, 054408 (2003).

${ }^{32}$ F. Bødker and S. Mørup, Europhys. Lett. 52, 217 (2000).

${ }^{33}$ S. Soeya, S. Nakamura, T. Imagawa, and S. Narishige, J. Appl. Phys. 77, $5838(1995)$

${ }^{34}$ T. Ambrose and C. L. Chien, J. Appl. Phys. 83, 6822 (1998). 